\title{
SOBRE LA ESTIMACIÓN DE LA TEMPERATURA DE LAS AGUAS MARINAS SUPERFICIALES DEL PLIOCENO A PARTIR DE ASOCIACIONES DE FORAMINÍFEROS PLANCTÓNICOS RECIENTES
}

\author{
José M. GONZÁLEZ-DONOSO, Francisco SERRANO \\ y Dolores LINARES
}

Departamento de Geología, Universidad de Málaga, 29071-Málaga

\begin{abstract}
González Donoso, J.M., Serrano, F. y Linares, D. 1999. Sobre la estimación de la temperatura de las aguas marinas superficiales del Plioceno a partir de asociaciones de foraminíferos planctónicos recientes. [On the estimation of Pliocene sea surface temperature from assemblages of Recent planktonic foraminifera]. Revista Española de Paleontología, n⿳o extr. Homenaje al Prof. J. Truyols, 89-96. ISSN 0213-6937.
\end{abstract}

\begin{abstract}
The possibilities of applying the modern analog technique to estimate the temperature of the surface waters during the Pliocene are analyzed, starting from a calibration data set made up of taphocoenoses of recent planktonic foraminifera and their corresponding winter and summer temperatures. To simulate the composition of the taphocoenoses of the Pliocene intervals, after making suppositions about the ecological equivalence between extinct species and morphotypes and the recent ones, it is necessary to remove the current species without equivalents in such intervals from the calibration data set. The temperatures of a representative subset of 53 samples have been estimated in order to evaluate five modifications of the calibration data set. The averages of the errors in the estimates are not very high and the number of samples with high error of estimate is not excessive as well. The estimates of the summer temperatures are more reliable than the winter ones.
\end{abstract}

Keywords: Planktonic foraminifera, Pliocene, modern analog technique, paleotemperatures.

\section{RESUMEN}

Se exploran las posibilidades de aplicar la técnica de los análogos modernos a la estimación de las temperaturas de las aguas superficiales del mar durante el Plioceno, partiendo de un conjunto de calibración formado por tafocenosis recientes de foraminíferos planctónicos y sus correspondientes temperaturas invernales y estivales. Para simular la composición de las tafocenosis de los intervalos bioestratigráficos pliocénicos, tras realizar suposiciones sobre la equivalencia ecológica de especies y morfotipos extintos con otros actuales, se han eliminado, en el conjunto de calibración, las especies actuales sin equivalentes en tales intervalos. Para evaluar las cinco modificaciones resultantes se han estimado las temperaturas de un subconjunto de 53 muestras, representativo de las diferentes asociaciones y temperaturas presentes en el conjunto de calibración. Los errores medios de las estimaciones no aumentan demasiado, ni tampoco es excesivo el número de muestras con altos errores de la estimación. Las estimaciones de temperaturas estivales son más fiables que las invernales.

Palabras clave: Foraminíferos planctónicos, Plioceno, técnica de análogos modernos, paleotemperaturas.

\section{INTRODUCCIÓN}

Si se acepta la suposición de que las especies y sus variaciones morfotípicas mantienen los mismos requerimientos medioambientales a lo largo de su existencia, es posible inferir las características físicoquímicas de los medios pretéritos a partir del reconocimiento de fósiles de organismos pertenecientes a especies con representantes vivos.

En el caso más elemental, la identificación de fósiles de un morfotipo o especie permite realizar la inferencia de que el sedimento que los contiene se depositó en un medio cuyas características físico-químicas estaban incluidas dentro del rango de condiciones en que vive la especie en la actualidad (si hay argumentos suficientes para suponer que el fósil es autóctono) o se nutrió con elementos procedentes de biocenosis desarrolladas en un medio de dichas características (si se trata de un fósil alóctono).

$\mathrm{Si}$ se trata de una especie frecuente, conocida la relación existente entre su abundancia relativa en los 
sedimentos actuales y los valores de una variable ambiental, es posible ir más allá y realizar una estimación numérica del valor de dicha variable, para el caso del ambiente correspondiente a una muestra fósil (ver, por ejemplo, Ericson, 1968).

Alternativamente, se puede estudiar la relación entre la variación ecofenotípica de una característica morfológica en una especie actual y los valores de una variable medioambiental, y utilizar tal relación para inferir el valor de la variable en el medio en que vivieron los representantes fósiles de la especie (ver, por ejemplo, Kennett, 1976).

En el caso de taxones que abundan en estado fósil, conocida la composición cuantitativa de la asociación de especies contenida en una tafocenosis, es posible inferir las condiciones medioambientales correspondientes al material sedimentario que la contiene (o al área de la que se nutrió) con mucha mayor precisión y fiabilidad. Para ello se parte del establecimiento de las relaciones existentes entre la composición de las asociaciones de organismos del taxón en cuestión en una serie de tafocenosis recién formadas y los valores de la variable ambiental que se desea estudiar, dentro de un universo de muestreo, el conjunto de calibración, mejor cuanto más numeroso, diverso y extenso geográficamente sea, siempre que en el área abarcada por el conjunto en cuestión no haya variación geográfica de los requisitos medioambientales de las especies del taxón considerado.

Ciertos grupos de micro y nanofósiles, que abundan lo suficiente como para que sus asociaciones sean estudiadas cuantitativamente, son el objeto preferente de esta clase de estudios: foraminíferos (especialmente los planctónicos), ostrácodos, nanoplancton calcáreo, radiolarios, pólenes y un etcétera no muy largo. De ellos, es posible que sean los foraminíferos planctónicos los que más atención han recibido al respecto, existiendo un amplio elenco de estudios sobre las técnicas numéricas utilizables, a partir de los estudios pioneros de Imbrie y Kipp (1971), sobre las funciones de transferencia y de Hutson (1980), sobre las técnicas basadas en el reconocimiento de análogos modernos.

En este trabajo vamos a explorar las posibilidades de aplicar tales técnicas numéricas a un intervalo, el Plioceno, en el cual se registran algunas extinciones y apariciones de especies, lo que determina que, durante la mayor parte del mismo, las asociaciones de foraminíferos planctónicos difieran cualitativamente de las actuales.

\section{SUPOSICIONES NECESARIAS EN LA RECONSTRUCCIÓN AMBIENTAL CON TÉCNICAS NUMERICAS}

La aplicación de una técnica numérica con los fines citados requiere la aceptación de una serie de suposiciones particulares, aparte de la inicialmente citada, unas comprobables hasta cierto punto y otras no. Sin entrar en detalles al respecto (véase González Donoso y Linares, 1998, para una exposición detallada), la primera y más general es que la composición de la asociación de organismos fósiles refleja las condiciones del área de la cual procede. Los problemas de esta suposición son evidentes, comenzando por el hecho de que los organismos que componen una asociación fósil pueden provenir de una porción de la biosfera de condiciones no uniformes. En el caso de los foraminíferos planctónicos esto es obvio, pues las distintas especies de estos organismos viven a diversas profundidades dentro de la columna de agua, e incluso algunos cambian de profundidad a lo largo de la ontogenia.

La segunda es que la técnica numérica permite, a partir de la composición de la asociación, estimar adecuadamente la condición ambiental.

Ambas suposiciones se pueden someter a prueba estudiando la relación entre la composición de las tafocenosis actuales y las condiciones de las áreas en que viven las biocenosis de las que se supone proceden.

La tercera es que las especies presentes en las asociaciones actuales son las mismas que existen en la fósil; si es evidente que esto no se cumple, hemos de recurrir a las suposiciones adicionales pertinentes.

En el caso de los foraminíferos planctónicos, las asociaciones observables a lo largo del Pleistoceno están compuestas por las especies que existen en la actualidad. Partiendo, por tanto, de las suposiciones indicadas, se puede utilizar la técnica pertinente, sin más. Pero a lo largo del Plioceno, aunque se pueda reconocer la mayoría de las especies actuales, algunas de éstas faltan, mientras que están presentes otras hoy extinguidas. La aplicación de las técnicas numéricas requiere, por tanto, soluciones especiales y suposiciones adicionales de distinta naturaleza (véase, al respecto, Thunell, 1979; Dowsett y Poore, 1990; Dowsett, 1991; Serrano et al., 1999).

a) Si una especie extinta ha sido sustituida por otra que se supone es su descendiente directo (sea el resultado de un cambio anagenético, sea por una cladogénesis seguida por la sustitución posterior de la especie primitiva por la descendiente), se puede trabajar con la suposición de que la especie fósil ocupaba el mismo nicho ecológico que la especie actual. Aceptando esta suposición, se pueden aplicar las técnicas numéricas a los intervalos pliocénicos en que, por ejemplo, en lugar de Globorotalia inflata (d'Orbigny) está presente su antecesor inmediato, Globorotalia bononiensis Dondi, o el antecesor de ésta, Globorotalia puncticulata (Deshayes) (sin descender aquí a la discusión de si es acertado o incluso posible diferenciar estas tres especies sucesivas). Sin embargo, la aplicación de esta suposición puede ser arriesgada. Por ejemplo, las formas del grupo de Globoturborotalita apertura (Cushman), son frecuentes en algunos horizontes del Plioceno (por ejemplo, en una muestra del ODP, Site 976, mar de Alborán, llegan a ocupar el $46 \%$ de la asociación). Su representante actual, Globoturborotalita rubescens (Hofker) no sobrepasa el $14,5 \%$ en las tafocenosis actuales del Atlántico Norte y el Mediterráneo.

b) En el caso de una especie extinta, sin presumible descendiente actual, pero que muestra convergencia morfológica con una especie actual, se puede utilizar la suposición de que la primera ocuparía el mismo nicho 
ecológico que la segunda. Aceptándola, se puede utilizar el conjunto de calibración actual, que contiene la especie Globorotalia hirsuta (d'Orbigny), para estimar las condiciones ambientales de las muestras pliocénicas que contienen Globorotalia margaritae Bolli y Bermúdez. Ciertamente esta suposición puede ser atrevida, según muestra el análisis de los isótopos estables de especies del Paleoceno previamente interpretadas ecológicamente según sus semejanzas morfológicas con especies actuales (Shackleton et al., 1985).

c) Si la especie fósil no puede interpretarse bajo ninguna de las dos suposiciones anteriores, lo más adecuado (aunque no sea muy satisfactorio) es no tenerla en cuenta a la hora de calcular la composición cuantitativa de la asociación. La suposición correspondiente es que la composición de la asociación, eliminada la especie fósil, es capaz de reflejar adecuadamente el valor de la variable ambiental. Esto implica, a su vez, que la especie a eliminar no interaccionaba, de manera importante, con otros componentes de la asociación, no afectando, por tanto, a las frecuencias absolutas de los mismos.

d) Si la especie actual no está representada en el intervalo pliocénico, caben dos alternativas:

- tratar la asociación fósil como si fuera una actual en la que no está presente la especie en cuestión (obviamente, esta opción puede producir falsas semejanzas entre muestras actuales y fósiles, si se trata de una especie actual abundante).

- recalcular las abundancias relativas de las especies en el conjunto de calibración tras eliminar dicha especie y restablecer las relaciones entre la composición de las asociaciones y la variable ambiental. Este proceder está justificado bajo la suposición (como en el caso $c$ ) de que la presencia de la especie en cuestión no afecta a la abundancia absoluta de las otras especies en las biocenosis en que coexisten.

Para someter a prueba la validez de las dos primeras hipótesis habría que recurrir a estimar por otros métodos las condiciones en que vivió la especie fósil. Los isótopos estables, pese a los conocidos problemas que conlleva la conversión de sus valores en estimaciones de las variables ambientales, parecen una buena opción al respecto. Otra alternativa podría ser la utilización paralela de otro grupo taxonómico. Pero, en cualquier caso, debe quedar claro que la no coincidencia de los resultados no garantizaría que la suposición respecto a la equiparación de la especie extinta es errónea; puede ocurrir que sea la técnica destinada a contrastar los resultados la que ha suministrado una estimación errónea. Respecto a la tercera hipótesis, habría que comprobar si la estimación del valor de la característica es satisfactoria, pudiendo en tal caso utilizarse las mismas comprobaciones que para las dos anteriores, y con las mismas reservas.

La segunda opción para solucionar el cuarto caso es susceptible de experimentación, realizando las modificaciones oportunas en el conjunto de calibración y comparando las estimaciones de los valores de la variable que se obtienen con y sin la modificación en muestras de tafocenosis actuales. En este trabajo vamos a desarrollar una serie de modificaciones encaminadas a este fin.

\section{MÉTODOS}

En un trabajo precedente (González Donoso y Linares, 1998) se evaluaron las estimaciones de las temperaturas invernales realizadas a partir de un conjunto de calibración que comprende muestras del Atlántico Norte y del Mediterráneo, utilizando una serie de funciones de transferencia y técnicas de análogos modernos. Los mejores resultados se obtuvieron con una técnica de análogos modernos, utilizando la distancia de la cuerda al cuadrado como coeficiente de disimilitud y el cuadrado de la inversa de la distancia como función de ponderación. Esta es la técnica que hemos aplicado en los experimentos realizados en el presente trabajo.

Como conjunto de calibración se utilizó el mismo que en el trabajo precedente. Ahora bien, para evitar errores difíciles de controlar, se procedió a eliminar las muestras cuya estimación, con la técnica citada, tenía un error mayor de $2{ }^{\circ} \mathrm{C}$ o menor de $-2{ }^{\circ} \mathrm{C}$. De este modo, el conjunto de calibración inicial de 605 muestras quedó reducido a 556. Como variables ambientales se escogieron dos, la temperatura invernal de las aguas superficiales, entendida como la media de los tres valores mensuales más bajos (para evitar los problemas de la inversión térmica ecuatorial; en el trabajo precedente se utilizó la media de los tres meses invernales) y la estival, correspondiente a la media de los tres valores más altos.

Como muestras a estimar se seleccionó una parte del conjunto de calibración, procurando que el lote tuviera la mayor diversidad posible en cuanto a asociaciones y temperaturas. Para lograr tal fin, el conjunto de calibración se dividió en cinco grupos, diferenciados en función de la composición de las asociaciones, utilizando el algoritmo de las k-medias; para confirmar las atribuciones a grupos se realizó un análisis discriminante. Los grupos así diferenciados (Linares et al., 1999) son oceanográficamente coherentes, mostrando poca superposición geográfica y corresponden a condiciones polares, subpolares, intermedias, subtropicales-tropicales y ecuatoriales, respectivamente. De cada uno de estos grupos se tomó una muestra de entre las comprendidas en un entorno de un grado de temperatura invernal. Así resultó un total de 53 muestras que, con sus correspondientes abundancias relativas de las especies, se muestran en la Tabla 1.

Para realizar cada modificación del conjunto de calibración, se eliminaron una o más especies y se recalcularon las abundancias relativas de las restantes. Tras proceder de la misma manera con las muestras seleccionadas, se volvió a aplicar la técnica de los análogos modernos citada, obteniéndose así la estimación correspondiente.

Siguiendo el uso normal, denominaremos error de una estimación a la diferencia entre el valor "real" de la variable, calculado en este caso a partir de los datos leídos directamente en los mapas de Gorshkov (1978) y el valor estimado de la variable. Dado que en este estado de la investigación interesa la magnitud pero no el signo del error, la exposición se centrará en los valores absolutos de los errores.

Vamos a denominar incremento del valor medio del 
$\mathrm{N}$ G BUL CAL CGL CRS DEH DIG DUT FAL GLT HRS INF CUL OBL PCD PCS QNQ RBC RUB TRL SCI SPH TEN TRD TRS TUM UNV \begin{tabular}{lllllllllllllllllllllllllllllllll}
\hline A & 0,000 & 0,000 & 0,000 & 0,000 & 0,000 & 0,000 & 0,000 & 0,000 & 0,000 & 0,000 & 0,000 & 0,000 & 0,000 & 0,021 & 0,976 & 0,000 & 0,000 & 0,000 & 0,000 & 0,000 & 0,000 & 0,000 & 0,000 & 0,000 & 0,000 & 0,000
\end{tabular} $\begin{array}{llllllllllllllllllllllllllllllllll}2 \text { A } & 0,003 & 0,000 & 0,000 & 0,000 & 0,000 & 0,000 & 0,000 & 0,000 & 0,000 & 0,000 & 0,000 & 0,000 & 0,000 & 0,013 & 0,984 & 0,000 & 0,000 & 0,000 & 0,000 & 0,000 & 0,000 & 0,000 & 0,000 & 0,000 & 0,000 & 0,000\end{array}$ $\begin{array}{llllllllllllllllllllllllllllll}3 & \text { A } & 0,000 & 0,000 & 0,000 & 0,000 & 0,000 & 0,000 & 0,000 & 0,006 & 0,003 & 0,000 & 0,000 & 0,000 & 0,000 & 0,031 & 0,954 & 0,003 & 0,000 & 0,000 & 0,000 & 0,000 & 0,000 & 0,000 & 0,000 & 0,000 & 0,000 & 0,000\end{array}$ $\begin{array}{llllllllllllllllllllllllllll}4 & \mathrm{~A} & 0,002 & 0,000 & 0,000 & 0,000 & 0,000 & 0,000 & 0,000 & 0,000 & 0,004 & 0,000 & 0,000 & 0,000 & 0,000 & 0,069 & 0,789 & 0,136 & 0,000 & 0,000 & 0,000 & 0,000 & 0,000 & 0,000 & 0,000 & 0,000 & 0,000 & 0,000\end{array}$ $\begin{array}{llllllllllllllllllllllllllll}5 & \mathrm{~A} & 0,180 & 0,000 & 0,000 & 0,000 & 0,000 & 0,000 & 0,000 & 0,000 & 0,074 & 0,000 & 0,002 & 0,000 & 0,000 & 0,127 & 0,369 & 0,225 & 0,000 & 0,000 & 0,000 & 0,000 & 0,000 & 0,000 & 0,000 & 0,000 & 0,000 & 0,000\end{array}$ $\begin{array}{lllllllllllllllllllllllllllll}6 \mathrm{~A} & 0,000 & 0,000 & 0,000 & 0,000 & 0,000 & 0,000 & 0,000 & 0,000 & 0,000 & 0,000 & 0,000 & 0,000 & 0,000 & 0,004 & 0,656 & 0,330 & 0,000 & 0,000 & 0,000 & 0,000 & 0,000 & 0,000 & 0,000 & 0,000 & 0,000 & 0,000\end{array}$

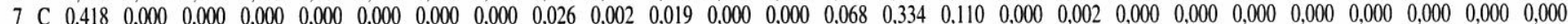

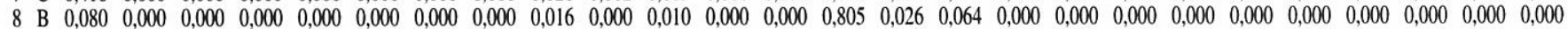
$\begin{array}{lllllllllllllllllllllllllllllll}9 & \mathrm{~A} & 0,068 & 0,000 & 0,000 & 0,000 & 0,000 & 0,000 & 0,001 & 0,000 & 0,017 & 0,000 & 0,003 & 0,000 & 0,000 & 0,308 & 0,479 & 0,125 & 0,000 & 0,000 & 0,000 & 0,000 & 0,000 & 0,000 & 0,000 & 0,000 & 0,000 & 0,000\end{array}$ $\begin{array}{llllllllllllllllllllllllllllll}10 & \mathrm{~B} & 0,144 & 0,000 & 0,000 & 0,000 & 0,000 & 0,000 & 0,000 & 0,000 & 0,036 & 0,000 & 0,007 & 0,000 & 0,000 & 0,727 & 0,022 & 0,065 & 0,000 & 0,000 & 0,000 & 0,000 & 0,000 & 0,000 & 0,000 & 0,000 & 0,000 & 0,000\end{array}$

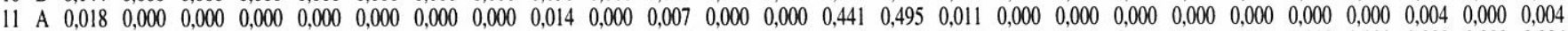

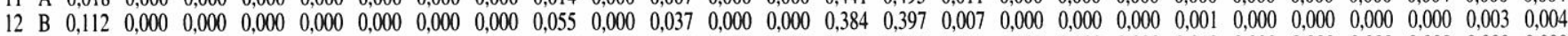
$\begin{array}{llllllllllllllllllllllllllll}13 & C & 0,447 & 0,000 & 0,000 & 0,000 & 0,000 & 0,000 & 0,000 & 0,000 & 0,066 & 0,000 & 0,030 & 0,000 & 0,000 & 0,179 & 0,060 & 0,205 & 0,000 & 0,000 & 0,000 & 0,013 & 0,000 & 0,000 & 0,000 & 0,000 & 0,000 & 0,000\end{array}$ $\begin{array}{llllllllllllllllllllllllllllll}14 & \text { B } & 0,249 & 0,000 & 0,000 & 0,000 & 0,000 & 0,000 & 0,000 & 0,000 & 0,232 & 0,000 & 0,036 & 0,000 & 0,000 & 0,426 & 0,000 & 0,033 & 0,000 & 0,000 & 0,000 & 0,011 & 0,000 & 0,000 & 0,000 & 0,000 & 0,000 & 0,000\end{array}$ $\begin{array}{lllllllllllllllllllllllllllllll}15 & \mathrm{~B} & 0,147 & 0,000 & 0,000 & 0,000 & 0,000 & 0,000 & 0,000 & 0,000 & 0,150 & 0,003 & 0,065 & 0,000 & 0,000 & 0,533 & 0,010 & 0,049 & 0,003 & 0,000 & 0,000 & 0,029 & 0,000 & 0,000 & 0,000 & 0,003 & 0,000 & 0,007\end{array}$ $\begin{array}{llllllllllllllllllllllllllllll}16 & \mathrm{~B} & 0,142 & 0,000 & 0,000 & 0,000 & 0,000 & 0,000 & 0,000 & 0,000 & 0,130 & 0,000 & 0,059 & 0,000 & 0,000 & 0,623 & 0,006 & 0,015 & 0,006 & 0,000 & 0,000 & 0,003 & 0,003 & 0,000 & 0,009 & 0,000 & 0,000 & 0,003\end{array}$ $\begin{array}{lllllllllllllllllllllllllllll}17 & \mathrm{E} & 0,084 & 0,003 & 0,000 & 0,000 & 0,000 & 0,000 & 0,542 & 0,009 & 0,046 & 0,000 & 0,136 & 0,003 & 0,006 & 0,136 & 0,000 & 0,015 & 0,000 & 0,000 & 0,000 & 0,000 & 0,000 & 0,000 & 0,000 & 0,000 & 0,000 & 0,000\end{array}$ $\begin{array}{lllllllllllllllllllllllllllll}18 & \mathrm{C} & 0,089 & 0,000 & 0,023 & 0,000 & 0,000 & 0,003 & 0,034 & 0,000 & 0,026 & 0,003 & 0,417 & 0,016 & 0,013 & 0,206 & 0,039 & 0,003 & 0,003 & 0,073 & 0,013 & 0,000 & 0,005 & 0,005 & 0,005 & 0,000 & 0,005 & 0,000\end{array}$ $\begin{array}{llllllllllllllllllllllllllllll}19 & \mathrm{~B} & 0,202 & 0,000 & 0,000 & 0,000 & 0,000 & 0,000 & 0,000 & 0,000 & 0,023 & 0,003 & 0,092 & 0,000 & 0,000 & 0,540 & 0,009 & 0,035 & 0,023 & 0,032 & 0,000 & 0,014 & 0,006 & 0,000 & 0,014 & 0,000 & 0,000 & 0,006\end{array}$ $\begin{array}{llllllllllllllllllllllllllllll}20 & \text { E } & 0,047 & 0,000 & 0,006 & 0,000 & 0,000 & 0,000 & 0,429 & 0,040 & 0,068 & 0,000 & 0,211 & 0,006 & 0,000 & 0,143 & 0,000 & 0,022 & 0,000 & 0,006 & 0,000 & 0,000 & 0,000 & 0,000 & 0,000 & 0,009 & 0,000 & 0,003\end{array}$ $\begin{array}{lllllllllllllllllllllllllllllll}21 & \mathrm{C} & 0,016 & 0,003 & 0,025 & 0,000 & 0,000 & 0,000 & 0,107 & 0,000 & 0,014 & 0,008 & 0,396 & 0,038 & 0,082 & 0,107 & 0,008 & 0,000 & 0,000 & 0,019 & 0,080 & 0,000 & 0,016 & 0,000 & 0,011 & 0,016 & 0,016 & 0,030\end{array}$ $\begin{array}{lllllllllllllllllllllllllllllll}22 & \mathrm{~B} & 0,113 & 0,002 & 0,002 & 0,000 & 0,000 & 0,002 & 0,000 & 0,006 & 0,089 & 0,012 & 0,204 & 0,000 & 0,000 & 0,377 & 0,077 & 0,026 & 0,000 & 0,008 & 0,000 & 0,004 & 0,004 & 0,000 & 0,032 & 0,008 & 0,000 & 0,010\end{array}$ $\begin{array}{llllllllllllllllllllllllllll}23 & \mathrm{D} & 0,101 & 0,000 & 0,000 & 0,000 & 0,000 & 0,000 & 0,000 & 0,146 & 0,022 & 0,000 & 0,093 & 0,000 & 0,000 & 0,086 & 0,000 & 0,019 & 0,000 & 0,399 & 0,030 & 0,000 & 0,019 & 0,000 & 0,000 & 0,037 & 0,000 & 0,049\end{array}$ $\begin{array}{llllllllllllllllllllllllllllll}24 & \mathrm{C} & 0,396 & 0,000 & 0,000 & 0,000 & 0,000 & 0,003 & 0,065 & 0,019 & 0,016 & 0,000 & 0,029 & 0,000 & 0,000 & 0,156 & 0,006 & 0,023 & 0,010 & 0,175 & 0,000 & 0,000 & 0,006 & 0,000 & 0,000 & 0,036 & 0,000 & 0,042\end{array}$ $\begin{array}{lllllllllllllllllllllllllllll}25 & \text { B } & 0,200 & 0,000 & 0,000 & 0,000 & 0,000 & 0,000 & 0,147 & 0,013 & 0,017 & 0,000 & 0,077 & 0,000 & 0,000 & 0,321 & 0,000 & 0,020 & 0,003 & 0,100 & 0,000 & 0,000 & 0,000 & 0,000 & 0,000 & 0,070 & 0,000 & 0,007\end{array}$

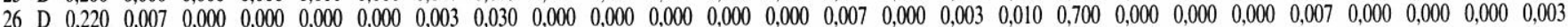
$\begin{array}{llllllllllllllllllllllllllll}27 & \mathrm{C} & 0,140 & 0,000 & 0,000 & 0,000 & 0,000 & 0,010 & 0,010 & 0,003 & 0,020 & 0,000 & 0,330 & 0,000 & 0,000 & 0,030 & 0,000 & 0,000 & 0,000 & 0,180 & 0,030 & 0,000 & 0,020 & 0,010 & 0,000 & 0,130 & 0,000 & 0,070\end{array}$ $\begin{array}{lllllllllllllllllllllllllllll}28 & \mathrm{~B} & 0,287 & 0,000 & 0,000 & 0,000 & 0,000 & 0,000 & 0,000 & 0,017 & 0,057 & 0,007 & 0,143 & 0,000 & 0,000 & 0,290 & 0,000 & 0,007 & 0,000 & 0,030 & 0,007 & 0,010 & 0,023 & 0,000 & 0,067 & 0,010 & 0,000 & 0,037\end{array}$ $\begin{array}{llllllllllllllllllllllllllllll}29 & \mathrm{D} & 0,106 & 0,000 & 0,000 & 0,000 & 0,000 & 0,013 & 0,008 & 0,031 & 0,004 & 0,000 & 0,000 & 0,000 & 0,000 & 0,009 & 0,013 & 0,000 & 0,031 & 0,681 & 0,000 & 0,000 & 0,040 & 0,004 & 0,000 & 0,000 & 0,000 & 0,049\end{array}$ $\begin{array}{lllllllllllllllllllllllllllll}30 & \mathrm{C} & 0,188 & 0,008 & 0,001 & 0,003 & 0,000 & 0,000 & 0,000 & 0,138 & 0,048 & 0,045 & 0,157 & 0,000 & 0,000 & 0,160 & 0,006 & 0,010 & 0,001 & 0,061 & 0,017 & 0,034 & 0,012 & 0,025 & 0,036 & 0,022 & 0,000 & 0,001\end{array}$ $\begin{array}{llllllllllllllllllllllllllllll}31 & C & 0,069 & 0,006 & 0,008 & 0,000 & 0,000 & 0,000 & 0,044 & 0,004 & 0,038 & 0,083 & 0,276 & 0,020 & 0,024 & 0,183 & 0,004 & 0,000 & 0,000 & 0,103 & 0,030 & 0,000 & 0,004 & 0,002 & 0,022 & 0,054 & 0,002 & 0,004\end{array}$ $\begin{array}{llllllllllllllllllllllllllll}32 & \mathrm{D} & 0,120 & 0,003 & 0,000 & 0,000 & 0,000 & 0,020 & 0,003 & 0,030 & 0,010 & 0,000 & 0,000 & 0,000 & 0,000 & 0,010 & 0,000 & 0,000 & 0,010 & 0,590 & 0,100 & 0,000 & 0,020 & 0,020 & 0,000 & 0,000 & 0,000 & 0,030\end{array}$ $\begin{array}{llllllllllllllllllllllllllllll}33 & \mathrm{D} & 0,060 & 0,005 & 0,000 & 0,000 & 0,000 & 0,030 & 0,000 & 0,020 & 0,010 & 0,000 & 0,000 & 0,000 & 0,000 & 0,000 & 0,000 & 0,000 & 0,040 & 0,650 & 0,100 & 0,000 & 0,060 & 0,020 & 0,000 & 0,000 & 0,000 & 0,020\end{array}$ $\begin{array}{lllllllllllllllllllllllllllllll}34 & \mathrm{C} & 0,135 & 0,006 & 0,028 & 0,003 & 0,000 & 0,003 & 0,030 & 0,003 & 0,141 & 0,017 & 0,077 & 0,014 & 0,028 & 0,122 & 0,003 & 0,000 & 0,008 & 0,155 & 0,072 & 0,003 & 0,061 & 0,000 & 0,019 & 0,050 & 0,011 & 0,022\end{array}$ $\begin{array}{llllllllllllllllllllllllllll}35 & C & 0,026 & 0,044 & 0,003 & 0,008 & 0,000 & 0,003 & 0,031 & 0,013 & 0,107 & 0,005 & 0,000 & 0,034 & 0,034 & 0,005 & 0,000 & 0,005 & 0,005 & 0,384 & 0,078 & 0,000 & 0,110 & 0,000 & 0,013 & 0,016 & 0,016 & 0,024\end{array}$ $\begin{array}{lllllllllllllllllllllllllllllll}36 & \mathrm{D} & 0,014 & 0,012 & 0,002 & 0,005 & 0,002 & 0,002 & 0,000 & 0,099 & 0,034 & 0,026 & 0,099 & 0,000 & 0,005 & 0,036 & 0,000 & 0,000 & 0,005 & 0,401 & 0,103 & 0,005 & 0,041 & 0,010 & 0,029 & 0,034 & 0,000 & 0,010\end{array}$ $\begin{array}{lllllllllllllllllllllllllllllll}37 & \mathrm{D} & 0,012 & 0,009 & 0,000 & 0,006 & 0,000 & 0,000 & 0,000 & 0,118 & 0,068 & 0,012 & 0,035 & 0,000 & 0,000 & 0,132 & 0,000 & 0,000 & 0,003 & 0,424 & 0,056 & 0,009 & 0,024 & 0,006 & 0,012 & 0,015 & 0,000 & 0,009\end{array}$ $\begin{array}{lllllllllllllllllllllllllllll}38 & \mathrm{C} & 0,064 & 0,006 & 0,005 & 0,003 & 0,000 & 0,000 & 0,010 & 0,077 & 0,077 & 0,034 & 0,228 & 0,005 & 0,005 & 0,040 & 0,000 & 0,000 & 0,000 & 0,302 & 0,018 & 0,002 & 0,014 & 0,003 & 0,014 & 0,064 & 0,000 & 0,006\end{array}$ $\begin{array}{llllllllllllllllllllllllllllllll}39 & \mathrm{C} & 0,046 & 0,000 & 0,014 & 0,011 & 0,000 & 0,003 & 0,000 & 0,023 & 0,085 & 0,009 & 0,171 & 0,006 & 0,003 & 0,028 & 0,000 & 0,000 & 0,003 & 0,302 & 0,111 & 0,000 & 0,040 & 0,000 & 0,046 & 0,091 & 0,000 & 0,006\end{array}$ $\begin{array}{lllllllllllllllllllllllllllllll}40 & \mathrm{D} & 0,136 & 0,000 & 0,010 & 0,000 & 0,000 & 0,002 & 0,017 & 0,005 & 0,022 & 0,037 & 0,079 & 0,000 & 0,002 & 0,002 & 0,000 & 0,000 & 0,000 & 0,496 & 0,022 & 0,000 & 0,027 & 0,002 & 0,022 & 0,079 & 0,000 & 0,012\end{array}$ $\begin{array}{lllllllllllllllllllllllllllllllll}41 & \mathrm{D} & 0,006 & 0,002 & 0,023 & 0,041 & 0,019 & 0,002 & 0,031 & 0,002 & 0,012 & 0,000 & 0,156 & 0,101 & 0,004 & 0,021 & 0,000 & 0,000 & 0,000 & 0,360 & 0,088 & 0,000 & 0,004 & 0,000 & 0,014 & 0,029 & 0,053 & 0,002\end{array}$ $\begin{array}{lllllllllllllllllllllllllllll}42 & \mathrm{C} & 0,109 & 0,000 & 0,000 & 0,003 & 0,000 & 0,000 & 0,003 & 0,000 & 0,028 & 0,003 & 0,156 & 0,112 & 0,003 & 0,150 & 0,000 & 0,000 & 0,059 & 0,199 & 0,115 & 0,028 & 0,012 & 0,000 & 0,009 & 0,003 & 0,003 & 0,003\end{array}$ $\begin{array}{lllllllllllllllllllllllllllll}43 & \mathrm{D} & 0,006 & 0,014 & 0,003 & 0,000 & 0,000 & 0,003 & 0,026 & 0,034 & 0,054 & 0,000 & 0,000 & 0,037 & 0,111 & 0,000 & 0,000 & 0,000 & 0,014 & 0,434 & 0,060 & 0,009 & 0,060 & 0,011 & 0,066 & 0,003 & 0,000 & 0,029\end{array}$ $\begin{array}{lllllllllllllllllllllllllllllll}44 & C & 0,051 & 0,000 & 0,015 & 0,006 & 0,000 & 0,003 & 0,009 & 0,021 & 0,054 & 0,000 & 0,072 & 0,012 & 0,000 & 0,108 & 0,000 & 0,000 & 0,039 & 0,260 & 0,156 & 0,000 & 0,033 & 0,000 & 0,090 & 0,021 & 0,000 & 0,051\end{array}$ $\begin{array}{llllllllllllllllllllllllllllllllllll}45 & \mathrm{E} & 0,002 & 0,000 & 0,000 & 0,019 & 0,002 & 0,023 & 0,218 & 0,008 & 0,031 & 0,000 & 0,072 & 0,154 & 0,010 & 0,066 & 0,004 & 0,000 & 0,004 & 0,107 & 0,107 & 0,006 & 0,012 & 0,000 & 0,000 & 0,002 & 0,123 & 0,008\end{array}$ $\begin{array}{lllllllllllllllllllllllllllll}46 & \mathrm{D} & 0,006 & 0,019 & 0,003 & 0,012 & 0,003 & 0,003 & 0,072 & 0,044 & 0,062 & 0,003 & 0,000 & 0,090 & 0,040 & 0,006 & 0,000 & 0,003 & 0,009 & 0,355 & 0,150 & 0,000 & 0,031 & 0,009 & 0,050 & 0,000 & 0,000 & 0,006\end{array}$ $\begin{array}{llllllllllllllllllllllllllllll}47 & \mathrm{E} & 0,002 & 0,000 & 0,014 & 0,006 & 0,012 & 0,006 & 0,117 & 0,020 & 0,042 & 0,000 & 0,018 & 0,166 & 0,042 & 0,125 & 0,000 & 0,000 & 0,000 & 0,119 & 0,129 & 0,000 & 0,038 & 0,002 & 0,002 & 0,000 & 0,107 & 0,022\end{array}$ $\begin{array}{llllllllllllllllllllllllllllll}48 & \mathrm{D} & 0,006 & 0,022 & 0,000 & 0,000 & 0,000 & 0,000 & 0,045 & 0,045 & 0,057 & 0,000 & 0,000 & 0,054 & 0,061 & 0,003 & 0,000 & 0,003 & 0,006 & 0,449 & 0,089 & 0,003 & 0,045 & 0,010 & 0,032 & 0,003 & 0,000 & 0,041\end{array}$ $\begin{array}{llllllllllllllllllllllllllllll}49 & \mathrm{D} & 0,000 & 0,013 & 0,012 & 0,004 & 0,000 & 0,000 & 0,004 & 0,004 & 0,139 & 0,000 & 0,004 & 0,000 & 0,000 & 0,000 & 0,000 & 0,000 & 0,021 & 0,588 & 0,064 & 0,004 & 0,042 & 0,033 & 0,021 & 0,002 & 0,000 & 0,012\end{array}$ $\begin{array}{lllllllllllllllllllllllllllllll}50 & \mathrm{E} & 0,000 & 0,000 & 0,000 & 0,000 & 0,011 & 0,000 & 0,078 & 0,000 & 0,007 & 0,000 & 0,007 & 0,007 & 0,022 & 0,007 & 0,000 & 0,000 & 0,000 & 0,000 & 0,011 & 0,000 & 0,000 & 0,000 & 0,000 & 0,000 & 0,840 & 0,007\end{array}$ $\begin{array}{llllllllllllllllllllllllllll}51 & \mathrm{D} & 0,000 & 0,011 & 0,017 & 0,006 & 0,008 & 0,006 & 0,103 & 0,000 & 0,044 & 0,000 & 0,000 & 0,063 & 0,013 & 0,000 & 0,000 & 0,000 & 0,004 & 0,418 & 0,221 & 0,002 & 0,040 & 0,002 & 0,004 & 0,000 & 0,010 & 0,013\end{array}$ $\begin{array}{lllllllllllllllllllllllllllll}52 & \text { E } & 0,060 & 0,000 & 0,006 & 0,020 & 0,010 & 0,000 & 0,240 & 0,000 & 0,000 & 0,000 & 0,006 & 0,130 & 0,020 & 0,000 & 0,000 & 0,000 & 0,000 & 0,070 & 0,309 & 0,000 & 0,000 & 0,000 & 0,020 & 0,000 & 0,110 & 0,000\end{array}$ $\begin{array}{lllllllllllllllllllllllllllll}53 & \mathrm{D} & 0,000 & 0,013 & 0,005 & 0,000 & 0,003 & 0,000 & 0,016 & 0,005 & 0,048 & 0,000 & 0,000 & 0,106 & 0,027 & 0,000 & 0,000 & 0,000 & 0,008 & 0,464 & 0,191 & 0,003 & 0,053 & 0,003 & 0,000 & 0,000 & 0,024 & 0,016\end{array}$

$\mathrm{N}$ : muestras ordenadas por temperaturas invernales; G: grupo al que pertenece la muestra (A: polar; B: subpolar; C: intermedio; D: subtropical-tropical; E: ecuatorial); BUL: G. bulloides; CAL: G. calida; CGL: G. conglobatus; CRS: G. crassaformis; DEH: S. dehiscens; DIG: G. digitata; DUT: N. dutertrei; FAL: G. falconensis; GLT: G. glutinata; HRS: G. hirsuta; INF: G. inflata; CUL: G. cultrata; OBL: P. obliquiloculata; PCD: N. pachyderma dextrorsa; PCS: N. pachyderma sinistrorsa; QNQ: T. quinqueloba; RBC: G. rubescens; RUB: G. ruber; TRL: G. trilobus; SCI: G. scitula; SPH: G. siphonifera; TEN: G. tenellus; TRD: G. truncatulinoides dextrorsa; TRS: G. truncatulinoides sinistrorsa; TUM: G. tumida; UNV: O. universa.

Tabla 1. Muestras seleccionadas: frecuencias de las especies y grupos a los que pertenecen.

error absoluto (abreviadamente, incremento del error) a la diferencia entre la media de los errores absolutos de las estimaciones obtenidas tras la modificación del conjunto de calibración y la media de los errores absolutos de las estimaciones realizadas con el conjunto de calibración sin modificar $\left(0,77^{\circ} \mathrm{C}\right.$ es el error absoluto medio inicial de las temperaturas invernales y $0,70^{\circ} \mathrm{C}$ el de las estivales).

Daremos arbitrariamente el calificativo "estimada mejor" o "estimada peor" a la muestra en la que la diferencia entre el error absoluto de la estimación inicial (esto es, con el conjunto de calibración sin modificar) y el error absoluto de la estimación tras la modificación del conjunto de calibración sea menor de $-0,5{ }^{\circ} \mathrm{C}$ o mayor de $+0,5^{\circ} \mathrm{C}$, respectivamente.

\section{RESULTADOS}

\section{MODIFICACIÓN 1}

Se suprime Globorotalia truncatulinoides (d'Orbigny). Esta modificación está destinada a simular las asociaciones del intervalo comprendido entre el BPA (biohorizonte de primera aparición) de Globorotalia crassaformis (Galloway y Wissler) y el BPA de $G$. 
truncatulinoides, esto es, la mayor parte del Plioceno superior. Se supone que el conjunto $G$. puncticulata - $G$. bononiensis - G. inflata es el equivalente ecológico de las G. inflata actuales.

G. truncatulinoides está presente en 420 de las 556 muestras incluidas en el conjunto de calibración pero sólo supera el $10 \%$ en 40 muestras. Su porcentaje máximo es del $20,5 \%$.

En las temperaturas invernales (Tabla 2) el incremento del error (Fig. 1) es de $0,19{ }^{\circ} \mathrm{C}$, una muestra es estimada mejor y cinco peor, todas con diferencias de errores de más de $1^{\circ} \mathrm{C}$, llegando una a $4,7{ }^{\circ} \mathrm{C}$. Hay que destacar que de las cuatro muestras con porcentajes de $G$. truncatulinoides superior al $10 \%$, sólo una es estimada peor. Por otra parte, tres de las muestras tienen estimaciones con errores absolutos de más de $2{ }^{\circ} \mathrm{C}$ (Fig. 2) (recuérdese que ninguna de las incluidas en el conjunto de calibración llega a esa cifra en la estimación realizada a partir del conjunto sin modificar), aunque sólo una tiene un error abultado $\left(-5,6^{\circ} \mathrm{C}\right)$.

Para las temperaturas estivales, el incremento del error es mucho menor, de sólo $0,03^{\circ} \mathrm{C}$. La temperatura es también mejor en un caso y peor en cuatro, pero sólo en una de estas últimas se supera la diferencia de $1{ }^{\circ} \mathrm{C}$, precisamente en la muestra con $4,7{ }^{\circ} \mathrm{C}$ de diferencia invernal, que ahora pasa a $2,7^{\circ} \mathrm{C}$. A esto hay que añadir que tres de las muestras estimadas peor lo son también en cuanto a temperaturas invernales. Por otra parte, tres de las muestras tienen errores absolutos de más de $2{ }^{\circ} \mathrm{C}$ pero dos de ellas también tenían errores de más de $2{ }^{\circ} \mathrm{C}$ en la estimación inicial (13 muestras del conjunto de calibración tienen errores absolutos de más de $2{ }^{\circ} \mathrm{C}$ en la estimación inicial y dos de ellas quedaron incluidas en el subconjunto seleccionado). La estimación peor tiene un error de $-3,4^{\circ} \mathrm{C}$.

A la vista de lo expuesto, las estimaciones de las temperaturas estivales correspondientes a este intervalo bioestratigráfico son más fiables que las invernales. Por otra parte, hay que contar con que las muestras con errores absolutos de la estimación de la temperatura invernal de más de $2{ }^{\circ} \mathrm{C}$, cuyo porcentaje puede ser del orden del $8 \%$ para las muestras posteriores al BPA de $G$. truncatulinoides (dando por válidas las suposiciones indicadas en González Donoso y Linares, 1998) pasarían a un porcentaje del orden del $13 \%$ para este intervalo. En el caso de las temperaturas estivales, se pasaría del $5 \%$ al $10 \%$. Ni que decir tiene que estos porcentajes sólo se ofrecen a título de orientación.

\section{MODIFICACIÓN 2}

Se suprimen G. truncatulinoides y G. crassaformis. De esta manera se intenta simular el intervalo entre el BPA de $G$. puncticulata y el BPA de $G$. crassaformis (parte alta del Plioceno inferior) siempre que se acepten dos suposiciones: a) que, como en el caso anterior, el conjunto $G$. puncticulata - G. bononiensis - $G$. inflata es el equivalente ecológico de las $G$. inflata actuales y b) que $G$. hirsuta es el equivalente ecológico actual de $G$. margaritae. G. crassaformis está presente en 234 de las 556 muestras y alcanza un porcentaje máximo del 7,1\%;

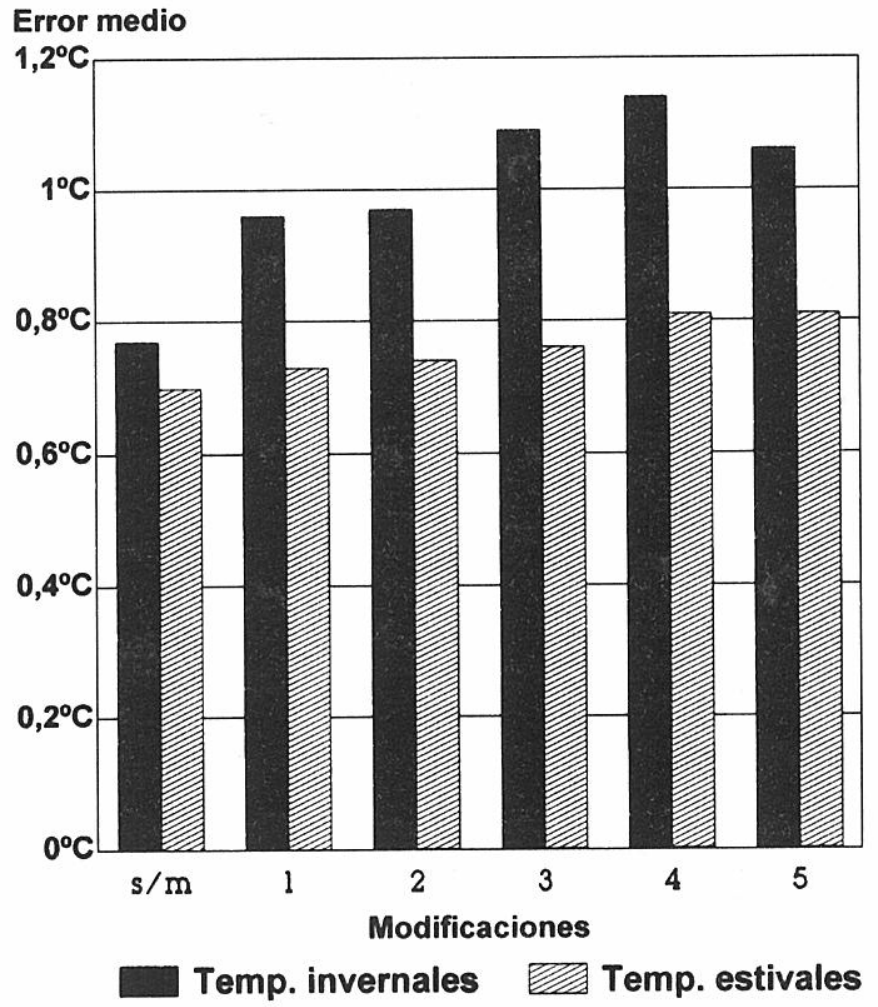

Figura 1. Valores medios de los errores absolutos de las estimaciones en cada modificación. $\mathrm{s} / \mathrm{m}$ : conjunto de calibración sin modificar.

sólo en 9 muestras excede el 3\% de la asociación.

En función de tales datos, no es previsible que su eliminación signifique grandes alteraciones de los resultados de la modificación anterior, pero se realizó también ésta, para mayor seguridad.

El incremento del error de las temperaturas invernales es de $0,20{ }^{\circ} \mathrm{C}$, casi idéntico al obtenido con la modificación anterior, y la diferencia máxima entre las estimaciones realizadas con las dos modificaciones es de $0,25^{\circ} \mathrm{C}$. Respecto a las muestras estimadas mejor o peor, sólo se incorpora una nueva muestra (que roza el valor arbitrario escogido de $-0,5{ }^{\circ} \mathrm{C}$ ) al grupo "peor"; las muestras con error absoluto de más de $2{ }^{\circ} \mathrm{C}$ son las mismas que en la modificación 1.

En cuanto a las temperaturas estivales, el incremento del error es de $0,04{ }^{\circ} \mathrm{C}$ y no hay ninguna variación respecto a la modificación anterior.

En función de lo expuesto, las consideraciones realizadas respecto al intervalo bioestratigráfico anteriormente tratado pueden ser extendidas a éste.

\section{MODIFICACIÓN 3}

Se suprimen $G$. truncatulinoides, G. crassaformis y $G$. hirsuta. Esta simulación sería la adecuada para el intervalo anterior si se supone que $G$. margaritae no es el equivalente ecológico de $G$. hirsuta, aunque sus morfologías presenten aspectos comunes. Esto, a su vez, obliga a eliminar $G$. margaritae en las muestras pliocénicas; el impacto de esta transformación en las estimaciones de las temperaturas pliocénicas no se puede evaluar directamente. G. hirsuta está representada en 309 


\begin{tabular}{|c|c|c|c|c|c|c|c|c|}
\hline \multirow[b]{2}{*}{$\mathrm{N}$} & & & \multicolumn{6}{|c|}{ TEMPERATURAS INVERNALES } \\
\hline & G & TL & TE0 & TE1 & TE2 & TE3 & TE4 & TE5 \\
\hline 1 & A & $-1,0$ & 0,1 & 0,0 & 0,0 & 0,0 & 0,0 & 0,0 \\
\hline & A & 0,0 & $-0,4$ & $-0,5$ & $-0,5$ & $-0,5$ & $-0,5$ & $-0,5$ \\
\hline 3 & A & 1,5 & 0,4 & $-0,1$ & $-0,1$ & $-0,1$ & $-0,1$ & $-0,1$ \\
\hline 4 & A & 3,0 & 4,9 & 4,9 & 4,9 & 4,9 & 5,0 & 5,0 \\
\hline & A & 4,0 & 5,7 & 5,6 & 5,6 & 5,6 & 5, & \\
\hline 6 & A & 4,8 & 3,7 & 3,7 & 3,7 & 3 , & 3,7 & 3,7 \\
\hline 7 & C & 5,0 & 5,6 & 5,3 & 5,3 & 5,3 & 5,3 & 5, \\
\hline 8 & B & $5 ; 0$ & 6,7 & 6,7 & 6,7 & 6,7 & 6,6 & 6, \\
\hline 9 & A & 6,0 & 5,7 & 5,7 & 5,7 & 5,7 & 5,7 & 5,7 \\
\hline 10 & B & 6,0 & 6,6 & 6,6 & 6,6 & 6,6 & 6,7 & 6,7 \\
\hline & A & 7,0 & 7,0 & 7,0 & 7,0 & 7 & 6,9 & 6,9 \\
\hline & B & 7,0 & 7,9 & 7,7 & 7,7 & 7 & 7,4 & \\
\hline & C & 7,7 & 7,2 & 7,2 & 7,3 & 7, & 7,1 & 7,1 \\
\hline & B & 8,0 & 8,5 & 8,5 & $-8,5$ & 8, & 8,6 & 8,5 \\
\hline & B & 9,0 & 9,8 & 9,8 & 9,8 & 9 & 9,9 & \\
\hline & B & 10,0 & 8,5 & 8,7 & 8,7 & 8,6 & 8,4 & 8,5 \\
\hline & E & 10,0 & 10,2 & 11,6 & 11,6 & $1 \mathrm{I}, 6$ & 11,6 & 11,6 \\
\hline & C & 10,3 & 9,3 & 13,3 & 13,3 & 15,5 & 13, & 11,7 \\
\hline & B & $11 ; 0$ & 11,4 & 11,1 & 11,1 & 11,1 & 11 , & 11,1 \\
\hline & E & 11 , & 9,8 & 10,6 & 10, & 10, & 10 , & 10,8 \\
\hline & $\mathrm{C}$ & & 13,3 & 13,0 & 13, & 13,4 & 19 , & 19,0 \\
\hline & B & 12, & 11,1 & 10,6 & & 10 , & 10 & 10,3 \\
\hline & D & 13, & 13,5 & 13,6 & 13 , & 13, & 13 & 3,8 \\
\hline & C & 13 & 13,3 & 13,4 & 13 , & 13 , & 13, & 13,5 \\
\hline & B & 13, & 13,1 & 12,7 & 12, & 12 & 12, & 12,5 \\
\hline & D & 14,0 & 15,1 & 15,1 & 15,1 & 15,1 & 15,1 & 15,1 \\
\hline & C & 14, & 11 & 14,1 & 14 , & 14 , & 4 , & 14,1 \\
\hline & B & 14, & 13,7 & 13, & 13, & 13, & 13 & 13,7 \\
\hline & D & & 5,1 & 15 , & 15,1 & 15,1 & 15,1 & 15,1 \\
\hline & C & & & & 14, & 14 & 1 & 14,0 \\
\hline & C & 16 & 15,8 & 14 & 14,0 & 12 & 14 & 15,4 \\
\hline & D & & & 16 & 16 , & 16 & 16 & 16,5 \\
\hline & D & & & & & & & \\
\hline & C & & 16,3 & 16,1 & 16,1 & 16 & 16,1 & 16,1 \\
\hline & C & & & & 23 & 22 & & 20,4 \\
\hline & D & & 19,9 & 19 & 19, & 19 & & 19,8 \\
\hline & D & & & & & & & 19,0 \\
\hline & C & & & & & & & 19,0 \\
\hline & C & & & 20 & 20 , & 20 & 20 & 20,1 \\
\hline & D & & & & 18 & 15 & & 17,8 \\
\hline & D & & & & 21 & & & \\
\hline & C & & & 21 & 21 , & 21 & 21 & \\
\hline & D & & & & & & & \\
\hline & C & & & 21 & 21 & & & \\
\hline & E & & & 24 & 24 & 24 & & \\
\hline & D & & & & 22 & 22 , & & \\
\hline & E & & & 25,0 & 25 , & 25,0 & & \\
\hline & D & & & 22,1 & 22,0 & 22,0 & & \\
\hline & D & & & 24,2 & 24,2 & 24,2 & 24,3 & \\
\hline & E & & & & 25 & & & \\
\hline & D & & & & 25 , & 25,4 & & \\
\hline & E & 26 & 25,7 & 25,7 & 25,7 & 25,7 & 25,7 & 25,7 \\
\hline & D & 27,0 & 26,8 & 26,7 & 26,8 & 26,8 & 26,8 & \\
\hline
\end{tabular}

\begin{tabular}{|c|c|c|c|c|c|c|}
\hline & \multicolumn{6}{|c|}{ TEMPERATURAS ESTIVALES } \\
\hline TL & TE0 & TE1 & TE2 & TE3 & TE4 & TES \\
\hline 5,5 & 6,5 & 6,5 & 6,5 & 6,5 & 6,5 & \\
\hline & 6,8 & 6,8 & 6,8 & & 6,8 & \\
\hline & 6,4 & 6,3 & 6,3 & 6,3 & 6,3 & \\
\hline 8, & 9,6 & 9,6 & 9,6 & 9,6 & 9,6 & \\
\hline 8,5 & 10,9 & 10,5 & 10,5 & 10,5 & 10,5 & 10 \\
\hline 9,5 & 8,6 & 8,6 & 8,6 & & 8,6 & \\
\hline 10.2 & 10,8 & 10,2 & 10,2 & 10,2 & 10,2 & \\
\hline & 12,6 & 12,6 & 12,6 & 12,6 & 12,7 & \\
\hline & 10,2 & 10,2 & 10 , & 10,2 & 10,2 & \\
\hline & 11,7 & 11,7 & 11, & 11, & 11,8 & \\
\hline & 12,0 & 12,0 & 12 , & 12 , & 11,5 & \\
\hline & 12,3 & 12,1 & & & 11,7 & \\
\hline & 11,8 & 11,8 & 11 , & 12 , & 11,7 & \\
\hline & 12,6 & 12,6 & 12 , & 12 , & 12,7 & 2, \\
\hline & 14,4 & 14 , & & 14, & 14,4 & \\
\hline & 13,4 & 13 , & 13 & 13,3 & 13 , & \\
\hline & 22,3 & & & & 22 , & \\
\hline & 20,7 & 22,8 & 22 , & 23 & 23 & \\
\hline & 17,8 & 17, & 17, & 17,7 & 17,7 & \\
\hline & 22,3 & & & 22,7 & & \\
\hline & 23,6 & 23 , & 23 , & 23,6 & 26,2 & \\
\hline & 16,4 & & & 15, & 15,8 & \\
\hline & 23,7 & 23 , & 23 & & & \\
\hline & 23,2 & 23 , & 23 , & 23, & 23,3 & \\
\hline & 2,4 & 2 & 22 , & & & \\
\hline & 4,1 & 2 & 24 , & 24, & 24 & \\
\hline & 3,5 & & & 23 & 23 & \\
\hline & 18,9 & 19 & 19 & 19 & 19 & \\
\hline & 24,4 & 24 & 24 & 24 & & \\
\hline & 0,4 & & & & & \\
\hline & 4,4 & 2 & 22 & 22 & & \\
\hline & 5,5 & & 25 , & 25 & 25 & \\
\hline & 5,1 & & & & & \\
\hline & 5,6 & & & 25 & & \\
\hline & & & & & & \\
\hline & 23,9 & & & & & \\
\hline & 2,4 & & 22 & 22 & & \\
\hline & 6,4 & & & & & \\
\hline & 4,4 & & & 24 & & \\
\hline & & & & & & \\
\hline & & & & & & \\
\hline & 5,9 & & & 25 & & \\
\hline & & & & & & \\
\hline & & & & & & \\
\hline & & & & 27 & 27 & \\
\hline & & & & & & \\
\hline & & 27 , & 27 & & & \\
\hline & & & & & & \\
\hline & & & & & & \\
\hline & & & & & & \\
\hline & & & & & & \\
\hline & & 27 & 27 & 27,9 & & \\
\hline & 21,5 & $2 /, 4$ & 27,4 & 21,4 & & \\
\hline
\end{tabular}

$\mathrm{N}$ : muestras ordenadas por temperaturas invernales; G: grupo al que pertenece cada muestra; TL: temperatura leída; TE0: temperatura calculada a partir del conjunto de calibración sin modificar; TE1: temperatura estimada eliminando G. truncatulinoides; TE2: temperatura estimada eliminando G. truncatulinoides y G. crassaformis; TE3: temperatura estimada eliminando G. truncatulinoides, G. crassaformis y G. hirsuta; TE4: temperatura estimada eliminando G. truncatulinoides, G. crassaformis, G. hirsuta y G. inflata; TE5: temperatura estimada eliminando G. truncatulinoides, G. crassaformis y G. inflata.

Tabla 2. Temperaturas resultantes de los análisis.

de las 556 muestras del conjunto de calibración, pero sólo en 33 supera el $10 \%$ de la asociación. Su porcentaje máximo es $24,1 \%$.

El incremento del error de las temperaturas invernales aumenta un poco respecto a la modificación anterior, hasta $0,32{ }^{\circ} \mathrm{C}$. Las muestras estimadas mejor y peor son las mismas, pero hay cuatro muestras con errores absolutos de más de $2{ }^{\circ} \mathrm{C}$, las tres anteriores y una nueva; además, tales errores son más altos, superando tres los 4 ${ }^{\circ} \mathrm{C}$ y llegando uno a $-5,2{ }^{\circ} \mathrm{C}$. Sin embargo, el porcentaje de $G$. hirsuta no supera el $5 \%$ en ninguna de las 53 muestras estimadas, y en la nueva -en cuanto a mala estimación- la especie está ausente.

Respecto a las temperaturas estivales, el incremento del error aumenta un poco, pasando a $0,06{ }^{\circ} \mathrm{C}$. Las muestras estimadas mejor y peor son las mismas y continúa habiendo tres muestras con errores absolutos mayores de $2{ }^{\circ} \mathrm{C}$, pero una es nueva, resultando que una de las muestras con error de la estimación inicial (conjunto sin modificar) mayor de $2{ }^{\circ} \mathrm{C}$ ahora es estimada con un error menor de $2{ }^{\circ} \mathrm{C}$. Ninguna de estas tres muestras llega a los $3{ }^{\circ} \mathrm{C}$ de error absoluto. 

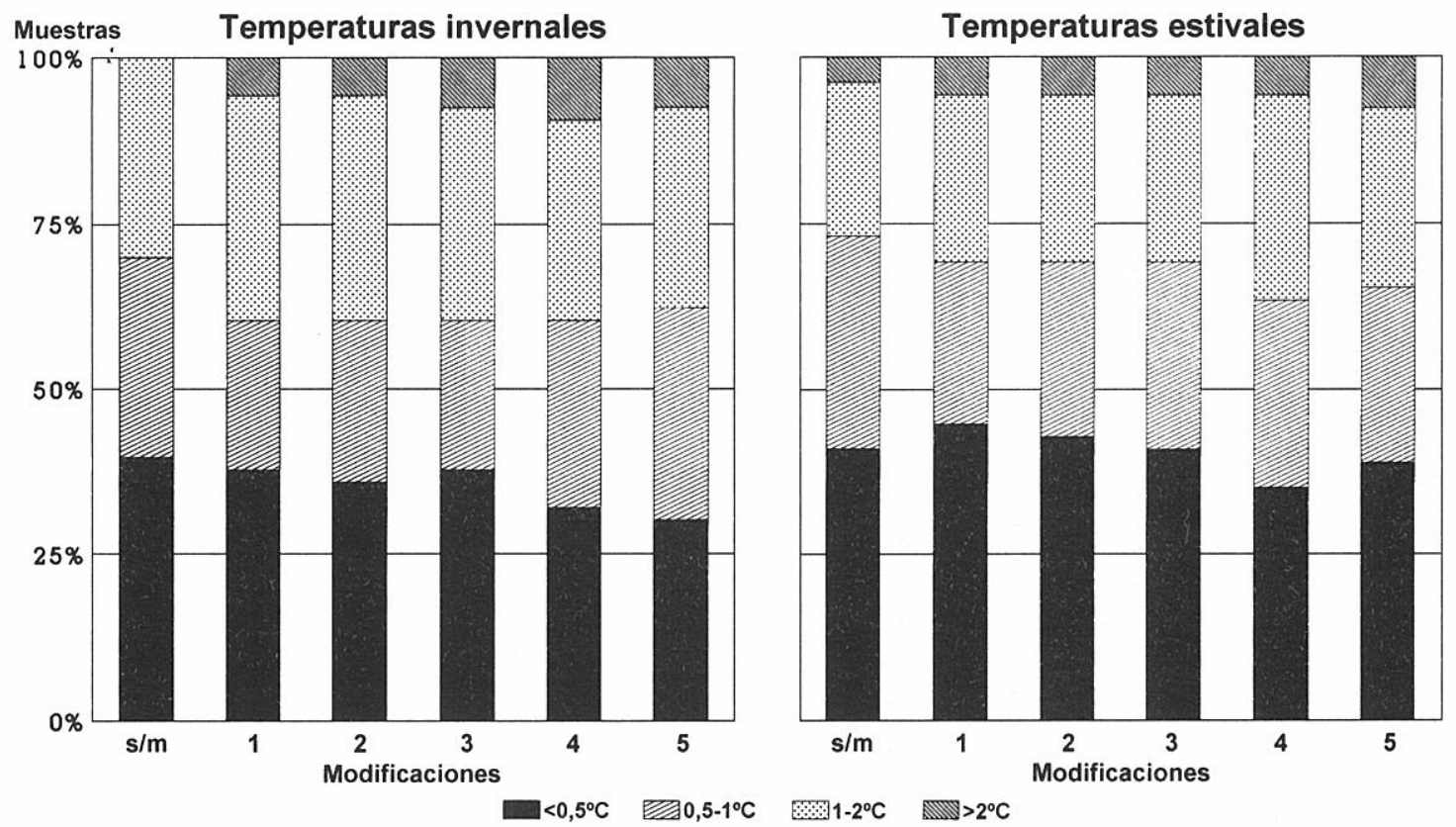

Figura 2. Errores absolutos clasificados por categorías para cada estimación.

Con todas las reservas ya expuestas, las muestras con estimaciones de las temperaturas invernales con más de 2 ${ }^{\circ} \mathrm{C}$ de error absoluto podrían estar en torno al $15 \%$ durante este intervalo. Las temperaturas estivales, que siguen siendo más fiables en cuanto a estimación, continúan con el porcentaje previsible de errores del orden del $10 \%$.

\section{MODIFICACIÓN 4}

Se suprimen $G$. truncatulinoides, G. crassaformis, $G$. hirsuta y $G$. inflata. Ahora se pretende simular el intervalo pliocénico anterior al BPA de G. puncticulata, (parte baja del Plioceno inferior) en la suposición de que $G$. hirsuta no es el equivalente ecológico actual de $G$. margaritae. $G$. inflata, la especie cuya eliminación marca la diferencia con la modificación anterior, está presente en 364 muestras del conjunto de calibración, supera el $10 \%$ de la asociación en 149 y el $25 \%$ en 37 muestras; su frecuencia máxima es de $41,7 \%$ siendo, por tanto, una especie "importante".

En lo que se refiere a las temperaturas invernales, el incremento del error se eleva a $0,37^{\circ} \mathrm{C}$. Hay una muestra con estimación mejor, la misma que en las modificaciones anteriores, y 8 con estimación peor, las seis anteriores y dos nuevas. En cuanto a muestras con error absoluto mayor de $2{ }^{\circ} \mathrm{C}$, hay cinco pero, salvo una con un error muy considerable $\left(-8,1^{\circ} \mathrm{C}\right)$, ninguna llega a los $4{ }^{\circ} \mathrm{C}$.

Tampoco se observa aquí una clara relación entre la abundancia de la nueva especie eliminada ( $G$. inflata en este caso) y el incremento (positivo o negativo) del error de la estimación. De las dos nuevas con estimación peor, una tiene casi un $40 \%$ de $G$. inflata en el conjunto de calibración sin modificar y es la que ostenta el error más grande $\left(-8,1{ }^{\circ} \mathrm{C}\right)$, pero la otra no llega al $16 \%$; una muestra con casi el $42 \%$ de $G$. inflata tiene un error de $-3,3^{\circ} \mathrm{C}$, mientras que en otra con un $33 \%$ de $G$. inflata el error es de $-0,1^{\circ} \mathrm{C}$.
Las temperaturas estivales tienen un incremento del error de $0,11{ }^{\circ} \mathrm{C}$ que, aunque pequeño, casi dobla al precedente. Hay dos muestras con estimación mejor, una de ellas la de las modificaciones anteriores, y seis con estimación peor, las cuatro anteriores y dos nuevas (las mismas que en el caso de las temperaturas invernales). Las muestras con error absoluto de la estimación mayor de $2{ }^{\circ} \mathrm{C}$ siguen siendo tres, dos que ya daban mal resultado en la estimación anterior y una nueva (la que tenía $-8,1^{\circ} \mathrm{C}$ de error en la temperatura invernal).

Es evidente que, dada la importancia de esta especie, que es el elemento característico de las asociaciones intermedias entre las subpolares y las subtropicalestropicales en el Atlántico Norte (Linares et al., 1999), era de esperar que tuviera una influencia mayor en los errores de la estimación. Los porcentajes esperables de muestras con errores absolutos mayores de $2^{\circ} \mathrm{C}$ son, como en el caso anterior, del $17 \%$ y del $10 \%$, respectivamente.

\section{MODIFICACIÓN 5}

Se suprimen $G$. truncatulinoides, G. crassaformis y $G$. inflata. En el supuesto de que G. margaritae sea el equivalente ecológico de $G$. hirsuta, esta modificación simularía el intervalo pliocénico anterior al BPA de $G$. puncticulata.

En teoría, los resultados deberían ser intermedios entre los de las modificaciones 3 y 4 , aunque más próximos a los de la 4 , por el peso de $G$. inflata, pero no lo son del todo. En lo que se refiere a las temperaturas invernales, el incremento del error es de $0,29{ }^{\circ} \mathrm{C}$, menor por tanto que en las modificaciones 3 y 4 . En cuanto a las muestras estimadas peor, son seis, como en la modificación 3, pero son las mismas de la 4, salvo dos que aparecían en las cuatro modificaciones previas. En cuanto a las muestras con error absoluto mayor de $2{ }^{\circ} \mathrm{C}$, hay cuatro, las mismas que en la modificación 4, menos una, la que precisamente tenía el error máximo en la modificación 3. 
Respecto a las estimaciones de temperaturas estivales, el incremento del error es de $0,11^{\circ} \mathrm{C}$, como en la modificación 4. En cuanto a estimación peor y errores mayores de $2{ }^{\circ} \mathrm{C}$, los resultados son idénticos también a los de la modificación 4 salvo que, casi en el valor límite, aparece otra muestra con menos de $-2{ }^{\circ} \mathrm{C}$.

En este caso, los porcentajes de muestras con errores absolutos mayores de $2{ }^{\circ} \mathrm{C}$ serían del orden del $15 \%$ y del $12 \%$, respectivamente.

\section{CONCLUSIONES}

Es evidente que la introducción de las modificaciones necesarias para estimar las temperaturas pliocénicas con técnicas numéricas, a partir de un conjunto de calibración actual, empeora las estimaciones obtenidas con el conjunto sin modificar (aunque unas pocas estimaciones puedan mejorar). Por otra parte, no existe una clara relación entre la abundancia relativa de la especie eliminada en una muestra y el error de la estimación que se comete. Pero para utilizar estas técnicas hay que asumir tales inconvenientes.

Por otra parte, la utilización de estas modificaciones destinadas a homogeneizar la composición cualitativa de las asociaciones actuales y pliocénicas no empeora excesivamente la media de los errores absolutos, pasándose, en el peor de los casos, de los $0,77^{\circ} \mathrm{C}$ de la estimación sin modificaciones a $1,14{ }^{\circ} \mathrm{C}$ para las temperaturas invernales y de $0,7{ }^{\circ} \mathrm{C}$ a $0,81{ }^{\circ} \mathrm{C}$ para las estivales.

En cuanto a las muestras mal estimadas (errores absolutos mayores de $2{ }^{\circ} \mathrm{C}$ ), en las temperaturas invernales se pasa de un $8 \%$ en el conjunto de calibración sin modificar, al $17 \%$ con la modificación de peores resultados, y del $5 \%$ al $12 \%$ en las temperaturas estivales. Los errores, puntualmente, pueden ser elevados, habiéndose detectado hasta $8,1^{\circ} \mathrm{C}$ en temperaturas invernales y $3,5^{\circ} \mathrm{C}$ en las estivales, pero las muestras que exhiben errores altos son muy escasas. Del total de 265 estimaciones de temperaturas invernales realizadas (53 para cada una de las modificaciones), sólo 16 superan los $3{ }^{\circ} \mathrm{C}$ de error absoluto; en el caso de las estivales, tal tipo de errores sólo se observan en 6 casos.

En fin, a la vista de nuestros resultados, las estimaciones de temperaturas estivales son más fiables que las invernales.

\section{AGRADECIMIENTOS}

Este trabajo se ha realizado en el marco de los proyectos PB90-797, PB93-0991 y PB97-1063 de la Dirección General de Investigación Científica y Técnica y los Grupos de Investigación RNM-146 y RNM-202 de la Junta de
Andalucía. Agradecemos al Prof. M. A. Lamolda sus valiosas sugerencias.

\section{BIBLIOGRAFÍA}

Dowsett, H.J. 1991. The development of a long-range foraminifer transfer function and application to Late Pleistocene North Atlantic climatic extremes. Paleoceanography, 6, 259-273.

Dowsett, H.J. and Poore, R.Z. 1990. A new planktic foraminifer transfer function for estimating PlioceneHolocene paleoeceanographic conditions in the North Atlantic. Marine Micropaleontology, 16, 1-23.

Ericson, D.B. 1968. Pleistocene climates and chronology in deep-sea sediments. Science, 162, 1227-1234.

González-Donoso, J. M. and Linares, D. 1998. Evaluation of some numerical techniques for determining paleotemperatures from planktonic foraminiferal assemblages. Revista Española de Paleontología, 13, 107-129.

Gorshkov, S.G. (Ed.) 1978. World Ocean Atlas. Volume 2. Atlantic and Indian Ocean. Pergamon Press, Oxford, 1305.

Hutson, W.H. 1980. The Agulhas Current during the Late Pleistocene, analysis of modern faunal analogs. Science, 207, 64-66.

Imbrie, J. and Kipp, N.G. 1971. A new micropaleontological method for quantitative paleoclimatology. Application to a late Pleistocene Caribbean core. In: The Late Cenozoic Glacial Ages (Ed. K.K. Turekian). Yale University Press, New Haven, 71- 131.

Kennett, J.P. 1976. Phenotypic variation in some Recent and Late Cenozoic planktonic foraminifera. In: Foraminifera, vol. 2 (Eds. R.H. Hedley and C.G. Adams). Academic Press, Londres, 111-170.

Linares, D., González-Donoso, J.M. and Serrano. F. 1999. Paleoceanographic conditions during the Quaternary at Sites 976 (Alboran Sea) and 975 (Menorca Rise) inferred from the planktonic foraminiferal assemblages: basis for a biostratigraphy. In: Ocean Drilling Program Leg 161, Scientific Results (Eds. M.C. Comas, R. Zahn, A. Klaus, et al.). Texas, 441-456.

Serrano, F., González-Donoso, J. M. and Linares, D. 1999. Biostratigraphy and paleoceanography of the Pliocene at Sites 975 (Menorca Rise) and 976 (Alboran Sea) from a quantitative analysis of the planktonic foraminiferal assemblages. In: Ocean Drilling Program Leg 161, Scientific Results (Eds. M.C. Comas, R. Zahn, A. Klaus, et al.). Texas, 185-196.

Shackleton, N. J., Corfield, R. M. and Hall, M.A. 1985. Stable isotope data and the ontogeny of Paleocene planktonic foraminifera. Journal of Foraminiferal Research, 15, 321-336.

Thunell, R.C. 1979. Pliocene-Pleistocene paleotemperature and paleosalinity history of the Mediterranean Sea, results from DSDP site 125 and 132. Marine Micropaleontology, 4, 173-187. 